\title{
Self-assembled silver nanowires as top electrode for poly(3,4ethylenedioxythiophene):poly(stylenesulfonate)/n-silicon solar cell
}

Ishwor Khatri*, Ayo Hoshino, Fumiya Watanabe, Qiming Liu, Ryo Ishikawa, Keiji Ueno and Hajime Shirai

The Graduate School of Science and Engineering, Saitama University, 255 ShimoOkubo, Sakuraku, Saitama 858-3676, Japan

\begin{abstract}
We prepare transparent, self-assembled polygonal silver nanowires (AgNWs) mesh by bubble template and transferred as top electrode in poly(3,4ethylenedioxythiophene):poly(stylenesulfonate) (PEDOT:PSS)/nsilicon hybrid solar cell, which shows power conversion efficiency of $10.62 \%$ under $100 \mathrm{~mW} / \mathrm{cm}^{2}$ of AM 1.5 illuminations. The self-assembled AgNWs mesh electrode exhibited a low sheet resistance $(50 \mathrm{Ohm} / \mathrm{square})$ with high transmittance $(87 \%)$ at a wavelength of $550 \mathrm{~nm}$. In self-assembled AgNWs mesh, AgNWs are well packed making continuous path for charge collection and transportation. Nevertheless, studies have also been carried out to transfer AgNWs from donor (glass or plastic) to receiver substrates (thermal release tape) and an expected reason for the transfer of $\mathrm{AgNW}$ from thermal release tape to the of PEDOT:PSS has also been proposed.
\end{abstract}

Keywords: silver nanowires mesh, hybrid solar cell, PEDOT:PSS 


\section{Introduction:}

Poly(3,4ethylenedioxythiophene):poly(stylenesulfonate) (PEDOT:PSS)/n-silicon hybrid solar cell has attracted great attention due to its high power conversion efficiency $(\eta)$ and solution processing at low temperature[1-13]. Several approaches like: optimization of interfacial layer formation $[5,6]$, three dimensional (3D) nanowires structures for light trapping and antireflecting properties [7-11], addition of guest materials [12-14], so far has been attempted to improve efficiency of the device. Furthermore, this type of solar cell reduces production cost of solar cell module due to the use of low quality silicon for the starting wafer, which is a major contribution component to the overall cell cost [15]. Simulation data has further projected that an ultimate efficiency of over $20 \%$ is possible with the band alignment of PEDOT:PSS and silicon by controlling interface states, surface reflection and other factors [16]. In this type of solar cell, the photocarriers are separated under the built-in potential at the interface of PEDOT:PSS/n-Si. Silicon generates photocarriers and transport electrons, while the organic layer (PEDOT:PSS) is used for hole transporting. Indium-tin oxide (ITO)[17], gold [8] or silver nanowires (AgNWs) [18] have been used as anode electrode. On the other hand, carbon nanotubes and graphene are also consider as an alternative top transparent electrode but their electrical conductivity is lower than metal nanowires [19]. Silver nanowires (AgNWs) exhibit high electrical conductivity and transparency, because silver has the lowest resistivity (as low as $1.59 \times 10^{-6}$ $\Omega . c m)$ among metals [20-27]. Silver is stable in pure air and water and, therefore, has high corrosion resistance. It has already been demonstrated that the device performance of the PEDOT:PSS/n-Si is not only limited by $\mathrm{n}-\mathrm{Si}[11]$ but also from charge collecting ability of the top electrode[8,28]. Therefore, the charge collection ability of top metal electrode is also an important parameter to improve the device performance.

In the present work, we demonstrate a solution free transfer process of selfassembled AgNWs mesh as top electrode in PEDOT:PSS/n-Si hybrid solar cell. A remarkable power conversion efficiency of $10.62 \%$ is achieved under $100 \mathrm{~mW} / \mathrm{cm}^{2}$ of AM 1.5G illumination. We believe that the improvement in the efficiency is due to the efficient charge collection and transportation by self-assembled AgNWs electrode. 


\section{Experimental details:}

AgNWs were prepared by polyol method[20-22]. After cleaning AgNWs in ethanol and water solution by repeated centrifugation process, it was dispersed in water that was bubbled with sodium dodecyl sulfate (SDS ) surfactant and laurly dimethylanine oxide (LDAO) thickening agent. When the bubbles containing the AgNWs were sandwiched between two glass substrates, the bubble ridges including the AgNWs formed continuous polygonal structures. Mesh structures were formed after air-drying, which was followed by heat-treatment at $200^{\circ} \mathrm{C}$ for $20 \mathrm{~min}$. The detail description about the fabrication process of self-assembled polygonal structure AgNWs mesh is given in ref.[21]. These polygonal mesh structures were transferred from glass substrate to the top layer of PEDOT:PSS/n-Si solar cell through thermal release tape (Nitto Denko). Solar cells were fabricated by following method. One-side-polished ntype CZ c-Si wafers $(3-5 \Omega \cdot \mathrm{cm}, 500 \mu \mathrm{m})$ with $(100)$ orientation was taken and cleaned by RCA1 and RCA2 processes. RCA1 process includes cleaning of the c-Si at solution of $\mathrm{NH}_{4} \mathrm{OH}: \mathrm{H}_{2} \mathrm{O}_{2}(5: 1)$ at $80{ }^{\circ} \mathrm{C}$ for 10 minutes followed by $\mathrm{HF}$ etching $(5 \%$, $1 \mathrm{~min})$. RCA2 process includes cleaning the RCA1 cleaned substrates at solution of $\mathrm{HCl}: \mathrm{H}_{2} \mathrm{O}_{2}(5: 1)$ at $80{ }^{\circ} \mathrm{C}$ for $10 \mathrm{~min}$. followed by $\mathrm{HF}$ etching $(5 \%, 1 \mathrm{~min})$. Finally, these substrates were washed in deionized water for $10 \mathrm{~min}$. PEDOT:PSS (Clevios PH1000) solution was prepared by adding 5\% dimethyl sulfoxide (DMSO), 50\% methanol and $0.1 \%$ zonyl surfactant. The solution was spin coated at $1000 \mathrm{rpm}$ on nSi substrate for $1 \mathrm{~min}$ followed by annealing at $140{ }^{\circ} \mathrm{C}$ for $30 \mathrm{~min}$. InGa alloy and $\mathrm{Ag}$ was used as bottom and top contact with $\mathrm{Ag} / \mathrm{PEDOT}: \mathrm{PSS} / \mathrm{n}-\mathrm{Si} / \mathrm{InGa}$ structure. In pristine device, $\mathrm{Ag}$ electrode was prepared by pattern printing, then an effective area of $5 \times 5 \mathrm{~mm}^{2}$ was defined by drawing a square shape Ag paste. For comprision, after transfering self-assembled AgNWs, an effective area of $5 \times 5 \mathrm{~mm}^{2}$ was also drawn in similar way. The outer area of the silver electrode was covered with an opaque mask to eliminate the incident light.

The current density-voltage $(\mathrm{J}-\mathrm{V})$ characteristics were measured in the dark and under illumination with simulated solar light (AM 1.5G, $100 \mathrm{~mW} / \mathrm{cm}^{2}$, Bunkoukeiki CEP-25BX). The calculation of $\eta$ was performed using the following equation: $\eta=V_{o c} J_{s c} F F / P_{\text {in }}$ where $V_{o c}$ is the open circuit voltage, $J_{s c}$ is the short circuit current density, FF is the fill factor, and $\mathrm{P}_{\text {in }}$ is the incident light power. FF is determined according to $F F=\left(V_{m} J_{m}\right) /\left(V_{o c} J_{s c}\right)$, where $V_{m}$ and $J_{m}$ are voltage and current density in 
the maximum power point of the J-V curves in the fourth quadrant. AgNWs mesh electrodes were characterized by scanning electronic microscopy (SEM; Hitachi S4100). SEM was performed at the operating voltage of $15 \mathrm{kV}$.

\section{Results and discussion:}

\subsection{Synthesis of AgNWs}

Silver nanowires (AgNWs) of about $10 \mu \mathrm{m}$ length and $100 \mathrm{~nm}$ diameter were prepared by controlling the concentration of poly(vinylpyrrolidone) (PVP) and silver nitrate $\left(\mathrm{AgNO}_{3}\right)$ in ethylene glycol(EG). EG acts as solvent and reductant, while PVP was used as the capping agent. In this process, silver nanoparticles were first produced due to the reduction of $\mathrm{AgNO}_{3}$ at $160^{\circ} \mathrm{C}$. These nanoparticles serve as seeds for the growth of silver nanorods. In second step, $\mathrm{AgNO}_{3}$ and PVP were added simultaneously. PVP prevents aggromolation of AgNPs due to chemically absorb macromolecules onto the surface of AgNPs. It also assists AgNPs to grow to rodshaped structure[20-22]. Figure 1 (a) shows the SEM image of as-synthesized AgNWs. These AgNWs were pentagonal shape[Fig 1 (b)] with $\{100\}$ facets on side surface and $\{111\}$ facets on end surface [22-26]. These AgNWs were used to parepared self-assembled AgNWs electrodes.

\subsection{Preparation of self-assembled AgNWs mesh electrode}

Self-assembled AgNWs mesh electrode was prepared using bubble template. Bubble template is useful because bubble templates do not need processes to remove the templates. To prepare bubble template with AgNWs, SDS and LDAO were added to AgNWs solution as described in experimental section. SDS and LDAO provide low surface tension and high viscosity, respectively. Fig. 2(a) shows bubbles that were formed by continuously stirring the AgNWs suspension. These bubbles were then sandwiched between two glass substrates as shown in Fig 2(b) and air dried for a few hours (2-3 hours). Fig 2(c) shows image of air-dried AgNWs mesh on glass substrates. AgNWs are self-assembled due to the draining flow[21 and therein]. The SEM image of AgNWs grid is shown in Fig 2(d). The inset figure suggest that AgNWs are well connected and make a continuous path. It has been observed that the line width and line height varied depending on the location. The line width varied from 2-30 $\mu \mathrm{m}$, whereas the line height varied from 2-15 $\mu \mathrm{m}$. After annealing at $200^{\circ} \mathrm{C}$ for $20 \mathrm{~min}$ [21], the sheet resistance of the mesh electrode was measured as 50 
Ohm/square with $87 \%$ transmittance at $550 \mathrm{~nm}$ wavelength. The sheet resistance of the mesh electrode can be further reduced with the use of single walled carbon nanotubes or graphene as suggested in ref $[29,30]$.

\subsection{Transfer of self-assembled AgNWs mesh}

Self-assembled AgNWs on glass substrates were transferred on the surface of PEDOT:PSS/n-Si solar cell. Fig. 3 shows the schematic diagram of transfer process. At first, a thermal release tape was pressed on the self-assembled AgNWs in glass substrate[Fig 3 (a)] and slowly released [Fig 3(b)], which was latter gently pressed on PEDOT:PSS/n-Si solar cell[Fig 3(c)]. The adhesive properties of tape disappear with mild heating (about $110^{\circ} \mathrm{C}$ for a few seconds), leaving AgNWs mesh on the surface of PEDOT:PSS layer. The device after transfer of self-assembled AgNWs is shown in Fig 3 (d). The outer area of the silver electrode was covered with an opaque mask to eliminate the incident light. Fig. 3(e) show the top view of the AgNWs mesh on PEDOT:PSS layer of hybrid solar cell.

Although, thermal release tape has been used to transfer the self-assembled AgNWs mesh, a complete transfer of self-assembled AgNWs from parent substrates (glass) couldn't be obtained. Fig. 4 (a) shows SEM image of self-assembled AgNWs on glass substrate after peeling off thermal release tape. The inset figure shows the parent substrate. The magnification portion of the marked spot in Fig 4(a) is shown in Fig 4(b). Many of the AgNWs were left on the substrate. However, the amount of AgNWs, which has been pelled off were enough to make a continuous path of selfassembled mesh on thermal release tape. Fig. 4(c) shows SEM image of the peeled off AgNWs from glass substrate on thermal release tape. The inset figure shows the thermal release tape. The magnification portion of the marked spot in Fig 4(c) is shown in Fig 4(d). The ridge of self-assembled AgNWs are continuous and connected with each other. SEM images on the same spots before and after peel off have been highlighted. Recently, C.H Lee et al. [31] also proposed similar approaches to transfer thin films of solar cell on universal substrates.

\subsection{Fabricate device.}

The devices were characterized with and without [Fig 5 (a)] self-assembled AgNWs as top electrode on PEDOT:PSS/n-Si solar cell. In this type of device, it is expected that photocarriers are separated under the built-in potential at the interface of PEDOT:PSS/n-Si. Silicon generates photocarriers and transport electrons, while the 
organic layer is used for hole transportation [9-13]. Charges are collected by metal electrodes deposited on the top $[\mathrm{Ag}]$ and bottom [ $\mathrm{InGa}]$ of the device. Current-voltage $(\mathrm{J}-\mathrm{V})$ characteristics were performed under illumination of AM $1.5 \mathrm{G} 100 \mathrm{~mW} / \mathrm{cm}^{2}$ simulated solar light. The device for pristine PEDOT:PSS buffer layer exhibited an $\eta$ of $10.32 \%$ with $\mathrm{J}_{\mathrm{sc}}$ of $30.80 \mathrm{~mA} / \mathrm{cm}^{2}$, a $\mathrm{V}_{\mathrm{oc}}$ of $0.542 \mathrm{~V}$, and a FF of 0.61 . Once selfassembled AgNWs grid was used as top electrode, $\eta$ increased to $10.62 \%$. with $\mathrm{J}_{\mathrm{sc}}$ of $29.31 \mathrm{~mA} / \mathrm{cm}^{2}, \mathrm{~V}_{\mathrm{oc}}$ of $0.537 \mathrm{~V}$ and $\mathrm{FF}$ of 0.67 . In particular, the FF value improved from 0.61 to 0.67 . Series resistance $\left(R_{s}\right)$ of the cell is the key parameter of the solar cell, which affects the FF. With self-assembled AgNWs electrode, the series resistance decreases from $1.57 \Omega . \mathrm{cm}^{2}$ to $0.95 \Omega . \mathrm{cm}^{2}$. The photovoltaic parameters $\left(\mathrm{J}_{\mathrm{sc}}\right.$, $\mathrm{V}_{\mathrm{oc}}, \mathrm{FF}$ and $\eta$ ) are summarized in Table1.

Self-assembled AgNWs were transferred on the surface of PEDOT:PSS and its photovoltaic performace were characterized. We believe that our PEDOT:PSS film might have been positively charged due to the phase segregation of PEDOT:PSS[3236], therefore AgNWs get transferred. DMSO, methanol and zonyl have been added to prepare PEDOT:PSS solution in order to improve the conductivity. Conductivity improve due to the expanded structure of PEDOT[32-34] or removable of excess amount of PSS[35,36], hence, increasing PEDOT concentration, which is positively charge material[37]. Therefore, the transfer of AgNWs might have been posible. However, further studies are needed to accept this assumption. Similar to this assumption, by depositing a monolayer of APTES molecules on glass substrate, Madaria et al. [38] could transfer randomly distributed AgNWs. They suggested that APTES molecules make the surface slightly positively charged because of the presence of $\mathrm{NH}_{2}$ groups, facilitating transfer of the nanowires[38-40].

\section{Conclusions:}

In summary, we transferred self-assembled AgNWs grid as top electrode for PEDOT:PSS/n-Si solar cell. A highly efficient c-Si/organic hybrid solar cell was obtained with power conversion efficiency of $10.62 \%$ with $\mathrm{J}_{\mathrm{sc}}$ of $29.31 \mathrm{~mA} / \mathrm{cm}^{2}, \mathrm{~V}_{\mathrm{oc}}$ of $0.537 \mathrm{~V}$ and FF of 0.67 . Such Si/organic hybrid cells can potentially further deliver high conversion efficiency combining with the textured structure.

Acknowledgements This study was partially supported by the Japan Science and Technology Agency (JST) Grant and the Grant-in-Aid for Scientific Research from the Ministry of Education, Culture, Sports, Science and Technology of Japan. 


\section{References}

[1] I. Khatri, Z. Tang, T. Hiate, Q. Liu, R. Ishikawa, K. Ueno and H. Shirai, Optical and carrier transport properties of graphene oxide based crystalline Si/organic Schottky junction solar cell, J. Appl. Phys. 114 (2013) 234506 (1-8).

[2] I. Khatri, Q. Liu, R. Ishikawa, K. Ueno, H. Shirai, Improved photovoltaic response by incorporating green tea modified multiwalled carbon nanotubes in organic/inorganic hybrid solar cell, Can. J. Phys. doi: 10.1139/cjp-2013-0506.

[3] S. Avasthi, S. Lee, Y-L. Loo and J. C. Sturm, Role of majority and minority carrier barriers silicon/organic hybrid heterojunction solar cells, Adv. Mater. 23 (2011) 5762-5766.

[4] Q. Liu, I. Khatri, R. Ishikawa, A. Fujimori, K.Ueno, K. Manabe, H. Nishino and H. Shiria, Improved photovoltaic performance of crystalline-Si/organic Schottky junction solar cells using ferroelectric polymers, Appl. Phys. Lett. 103 (2013) 163503 (1-4).

[5] F. Zhang, B. Sun, T. Song, X. Shu and S. Lee, Air stable, efficient hybrid photovoltaic devices based on poly(3-hexylthiophene) and silicon nanostructures, Chem. Mater, 23 (2011) 2084-2090.

[6] L. He, C. Jiang, H. Wng, D. Lai and Rashi, High efficiency planar Si/organic heterojunction hybrid solar cell Appl.Phys. Lett. 100 (2012) 073503 (1-3).

[7] X. Shen, B. Sun, D. Liu and S-T. Lee, Hybrid heterojunction solar cell based on organic-inorganic silicon nanowire array architecture, J. Am. Chem. Soc. 133 (2011) 19408-19415.

[8] S. Jeong, E.C. Garnett, S. Wang, Z. Yu, S. Fan, M.L. Brongersma, M.C. McGehee and Y.Cu, Hybrid silicon nanocone-polymer solar cells, Nano Lett. 12 (2012) 2971-2976.

[9] F. Zhang, T. Song and B. Sun, Conjugated polymer-silicon nanowire array hybrid schottky diode for solar cell application, Nanotechnology 23 (2012) 194006 (1-9).

[10] W. Lu, C. Wang, W. Yue and L. Chen, Si/PEDOT:PSS core/shell nanowire arrays for efficient hybrid solar cells, Nanoscale, 3 (2011) 3631-3634.

[11] F. Zhang, X. Hau, S.T. Lee, B. Sun, Heterojunction with organic thin layer for three dimensional high performance hybrid solar cells, J. Mater. Chem. 22 (2012) 5362-5368.

[11] L. He, C. Jiang, H. Wang, D. Lai and Rushi, Si nanowires organic semiconductor hybrid heterojunction solar cells towards 10\% efficiency, ACS. Mater. Interfaces, 4 (2012) 1704-1708.

[12] I. Khatri, T. Imamura, A. Uehara, R. Ishikawa, K. Ueno and H. Shirai, Chemical mist deposition of graphene oxide and PEDOT:PSS films for crystalline Si/organic heterojunction solar cells, Phys. Status Solidi C. 9 (2012) 2134-2137.

[13] I. Khatri, Z. Tang, Q. Liu, R. Ishikawa, K. Ueno, H. Shirai, Green-tea modified multiwalled carbon nanotubes for efficient poly(3,4-ethylenedioxythiophene):poly(stylenesulfonate(/n-silicon hybrid solar cell, Appl. Phys. Lett. 102 (2013) 063508 (1-5).

[14] I. Khatri, S. Adhikari, H.R. Aryal, T. Soga, T. Jimbo, M. Umeno, Improving photovoltaic properties by incorporating both single walled carbon nanotubes and functionalized multiwalled carbon nanotubes, Appl. Phys. Lett. 94 (2009) 093509 (1-3).

[15] E.C. Garnett, C. Peters, M. Brongersma, Y. Cui, M. McGehee IEEE, 2010 Photovoltaic Specialists Conf. (PVSC),Hawaii (Hawaii: IEEE) pp 000934-8.

[16] T.G. Chen, B.U. Huang, E.C. Chen, P. Yu, H.F. Meng, Micro-textured conductive polymer/silicon heterojunction photovoltaic devices with high efficiency, Appl. Phys. Lett. 101 (2012) $033301(1-5)$.

[17] S.C. Shiu, J.J. Chao, S. C. Hung, C. L. Yeh, C. F. Lin, Morphology dependence of silicon nanowire/poly(3,4-ethylenedioxythiophene):poly(styrenesulfonate)heterojunction solar cell, Chem. Mater. 22 (2010) 3108-3113.

[18] T.G. Chen, B.Y. Huang, H.W.Liu, Y.Y.Huang, H.T.Pan,H.F.Meng, P.Yu, Flexible silver nanowire meshes for high efficiency microtextured organic-silicon hybrid photovoltaic, ACS Appl. Mater. Interfaces, 4 (2012) 6857-6864.

[19] A. Kumar, C. Zhou, The race to replace tin-doped indium oxide: Which material will win?, ACS Nano, 4 (2010) 11-14,

[20] I. Khatri, J. Bao, N. Kishi, T. Soga, Similar device architectures for inverted organic solar cell and laminated solid-state dye sensitized solar cell, ISRN Electronics, 2012 (2012) 180787(1-10).

[21] T. Tokuno, M. Nogi, J. Jiu, T. Sagahara, K. Suganuma, Transparent electrodes fabricated via the self-assembly of silver nanowires using a bubble template, Langmuir, 28 (2012) 9298-9302.

[22] Y. Sun, B. Mayers, T. Herricks, Y. Xia, Polyol synthesis of uniform silver nanowires: A plausible growth mechanism and the supporting evidence, Nano Lett. 3 (2002) 955-960.

[23] T. Tokuno, M. Nogi, M. Karakawa, J. Jiu, T.T. Nge, Y. Aso, K. Suganuma, Fabrication of silver nanowire transparent electrodes at room temperature, Nano Rec. 4 (2011) 1215-1222.

[24] L.B. Hu, H.S.Kim, J.Y. Lee, P. Peumans, Y. Cui, Scalable coating and properties of transparent, flexible, silver nanowire electrodes, ACS Nano, 4 (2010) 2955-2963.

[25] Z.B. Yu, Q.W. Zhang, L. Li, Q. Chen, X.F. Niu, J. Liu, Q.B. Pei, Highly flexible silver nanowire electrodes for shape-memory polymer light-emitting diodes, Adv. Mater, 23 (2011) 664-668. 
[26] R. Zhu, C. H. Chung, K.C. Cha, W. Yang, Y.B. Zheng, H. Zhou, T.B.Song, C.C.Chen,P.S.Weiss, G.Li, Y.Yang, Fused silver nanowires with metal oxide nanoparticles and organic polymers for highly transparent conductors, ACS Nano, 5 (2011) 9877-9882.

[27] W.Gaynor, J. Y. Lee, P. Peumans, Fully solution-processed inverted polymer solar cell with laminated nanowire electrodes, ACS Nano, 4 (2010) 30-34.

[28] Y. Tsunomura, Y. Yoshimine, M. Taguchi, T. Baba, T. Kinoshita, H.Kanno, H. Sakata, E. Maruyama, M. Tanaka, Twenty-two percent efficiency HIT solar cell, Sol. Energy Mater. \& Solar Cell. 93 (2009) 670-673.

[29] Y. Wu, X. Zhang, J. Jie, C. Xie, X. Zhang, B. Sun, Y. Wang, P. Gao, Graphene transparent conductive electrodes for highly efficient silicon nanostructures-based hybrid heterojunction solar cells J. Phys. Chem. C, 117 (2013) 11968-11976.

[30] T. Tokuno, M. Nogi, J. Jiu, K. Suganuma, Hybrid transparent electrodes of silver nanowires and carbon nanotubes: a low-temperature solution process, Nano. Rec. Lett, 7 (2012) 281 (1-7).

[31] C. H. Lee, D. R. Kim, I. S. Cho, N. William, Q. Wang, X. Zheng, Peel-and-Stick:Fabricating thin film solar cell on universal substrates, Scienctific reports, 2 (2012) 1000 (1-4)

[32] Y. Xia, J. Ouyang, Highly conductive PEDOT:PSS films prepared through a treatment with geminal diols or amphiphilic fluoro compounds, Organic electronics 13 (2012) 1785-1792.

[33] Y. Xia, J. Ouyang, PEDOT:PSS films with significantly enhanced conductivities induced by preferential solvation with cosolvents and their application in polymer photovoltaic cells, J. Mater. Chem. 21 (2011) 4927-4936.

[34] Y. Xia, K. Sun, J. Ouyang, Highly conductive poly $(3,4-$ ethylenedioxythiophene):poly(styrenesulfonate) films treated with an amphiphilic fluoro compound as the transparent electrode of polymer solar cell, Energy. Environ. Sci, 5 (2012) 5325-5332.

[35] X. Crispin, F.L.E. Jakobsson, A. Crispin, P.C.M. Grim, P. Andersson, A. Volodin, C. V.

Haesendonck, M. V. Auweraer, W.R. Salaneck, M. Berggren, The origin of the high conductivity of poly(3,4-ethylenedioxythiophene)-poly(styrenesulfonate) (PEDOT-PSS) plastic electrodes, Chem. Mater. 18 (2006) 4354.

[36] A. Onorato, M.A. Invernale, I.D. Berghorn, C.Pavlik, G.A. Sotzing, M.B. Smith, Enhanced conductivity in sorbitol-treated PEDOT-PSS. Observation of an in situ cyclodehydration reaction, Synthetic Metals, 160 (2010) 2284-2289.

[37] S. Liu, J. Tian, L. Wang, Y. Luo, X. Sun, Production of stable aqueous dispersion of poly (3,4ethylenedioxythiophene) nanorods using graphene oxide as a stabilizing agent and their application for nitrite detection, Analyst 136 (2011) 4898-4902.

[38] A. R. Madaria, A. Kumar, F. N. Ishikawa, C. Zhou, Uniform, highly conductive, and patterned transparent films of a percolating silver nanowire network on rigid and flexible substrates using a dry transfer technique, Nano Rec, 3 (2010) 564-573.

[39] D. B. Hines, S. Mezhenny, M. Breban, E.D. Williams, V.W. Ballarotto, G. Esen, A. Southard,

M.S. Fugrer, Nanotransfer printing of organic and carbon nanotube thin-film transistors on plastic substrates, Appl. Phys. Lett. 86 (2005) 163101 (1-3).

[40] R.G. Freeman, K.C. Graber, K.J. Allison, R.M. Bright, J.A. Davis, A.P. Guthrie, M.B.Hommer, M.A. Jackson, P.C. Smith, D.G. Walter, M.J. Natan, Self-assembled metal colloid monolayers:An approach to SERS substrates, Science 267 (1995) 1629-1632.

\section{Figure caption}

Figure 1. SEM images of (a) as-synthesized AgNWs by polyol process. (b) AgNWs with pentagonal structure and $\{111\}$ facets at end structure (marked with arrow).

Figure 2. Photographs of (a) bubbles containing the AgNWs in a case. (b) bubbles sandwiched between two glass substrates. (c) AgNWs mesh structures on glass substrate (d) SEM image of selfassembled AgNWs. Inset figure shows the well connected ridge of AgNWs mesh.

Figure 3. Transferring process of self-assembled AgNWs mesh electrode through thermal release tape (a) Pressing adhesive tape on the selfassembled AgNWs mesh electrode (b) releasing the adhesive tape (c) pressing the self-assembled AgNWs contained adhesive tape on 
PEDOT:PSS/n-Si solar cell (d) photograph of self-assembled AgNWs on PEDOT:PSS surface (e) SEM image of top view of AgNWs on PEDOT:PSS

film.

Figure 4. SEM image of self-assembled AgNWs (a) on glass substrate, Inset figure shows photograph of parent substrate (b) magnified SEM image of marked portion (c) on thermal release tape. Inset figure show photograph of thermal release tape (d) magnified SEM image of marked portion

Figure 5 (a) Device structure of Ag/PEDOT:PSS/n-Si/InGa(a) J-V curves of c-Si heterojunction solar cell for PEDOT:PSS composite buffer layers with and without self-assembled AgNWs electrode.

Table 1. Photovoltaic characteristics of solar cells under A.M 1.5 simulated solar radiation.

\begin{tabular}{|l|c|c|l|l|l|l|}
\hline Device structures & $\begin{array}{c}\mathrm{J}_{\text {sc }} \\
\left(\mathrm{mA} / \mathrm{cm}^{2}\right)\end{array}$ & $\begin{array}{l}\mathrm{V}_{\text {oc }} \\
(\mathrm{V})\end{array}$ & $\mathrm{FF}$ & $\begin{array}{l}\eta_{1} \\
(\%)\end{array}$ & $\begin{array}{l}\mathrm{R}_{\mathrm{s}}{ }^{2} \\
(\Omega . \mathrm{cm})\end{array}$ & $\begin{array}{l}\mathrm{R}_{\text {sh }}{ }^{2} \\
\left(\mathrm{~K} \Omega . \mathrm{cm}^{2}\right)\end{array}$ \\
\hline n-Si/PEDOT:PSS & 30.80 & 0.54 & 0.61 & 10.32 & 1.57 & 3.29 \\
\hline $\begin{array}{l}\text { n-Si/PEDOT:PSS/self- } \\
\text { assembled AgNWs }\end{array}$ & 29.31 & 0.53 & 0.67 & 10.62 & 0.95 & 4.72 \\
\hline
\end{tabular}

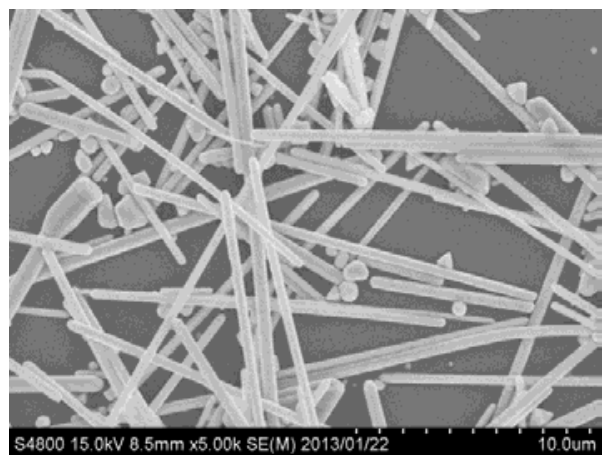

Figure 1

(a)

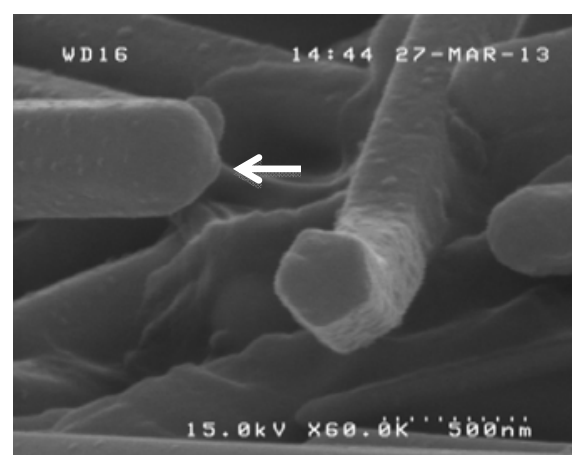

(b) 
Figure 2

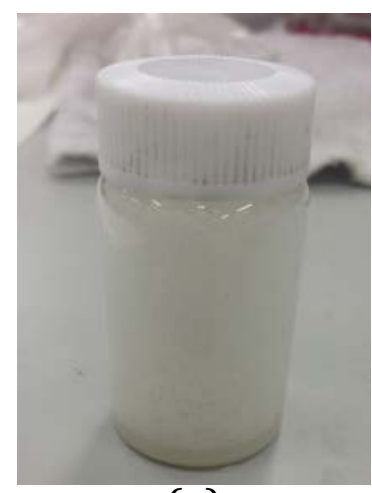

(a)

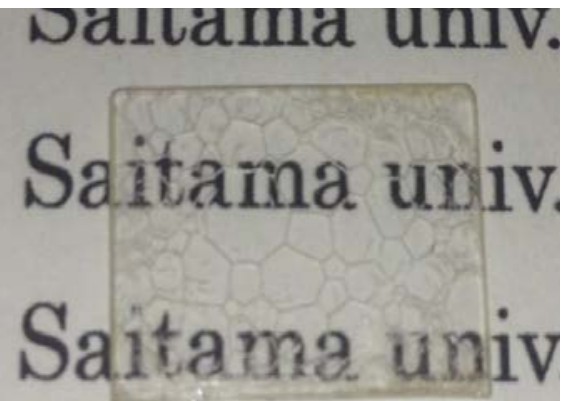

(c)

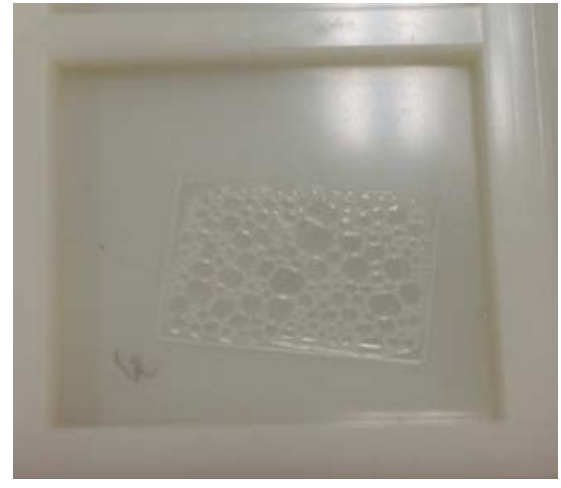

(b)

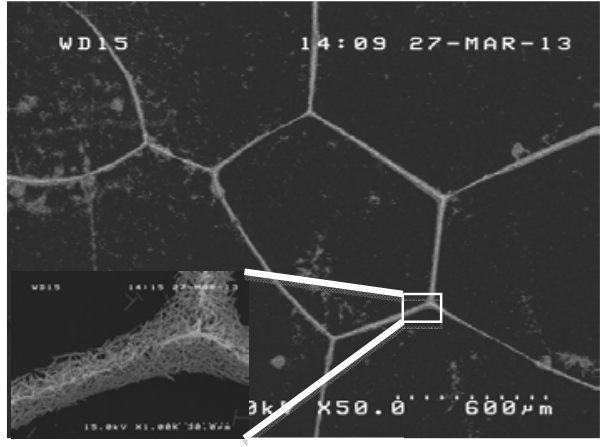

(d) 
Figure 3

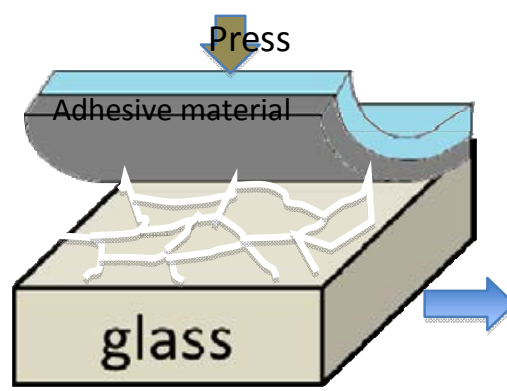

(a)

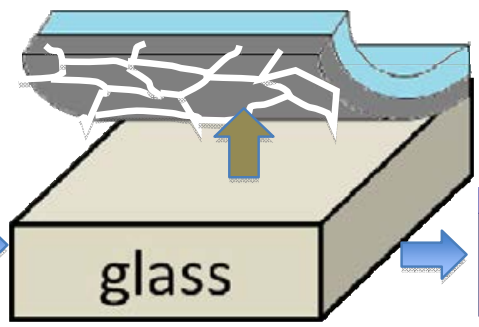

(b)

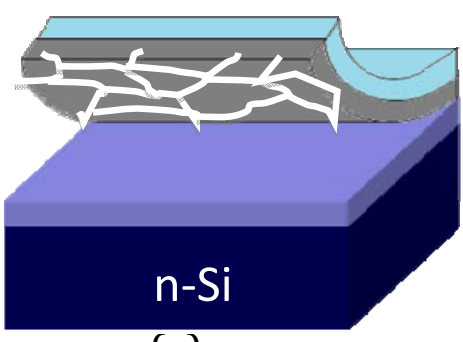

(c)

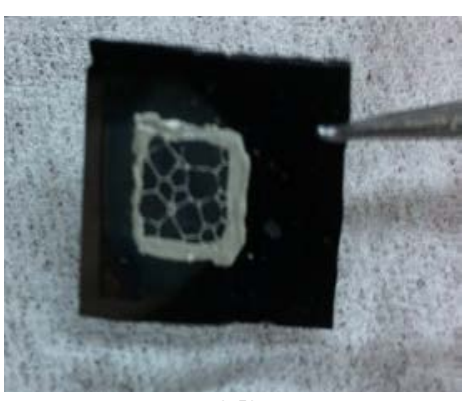

(d)

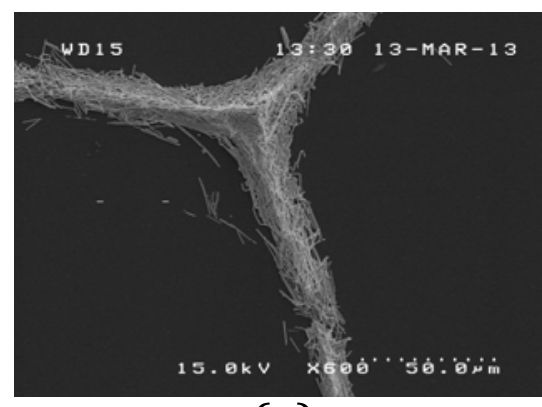

(e) 
Figure 4

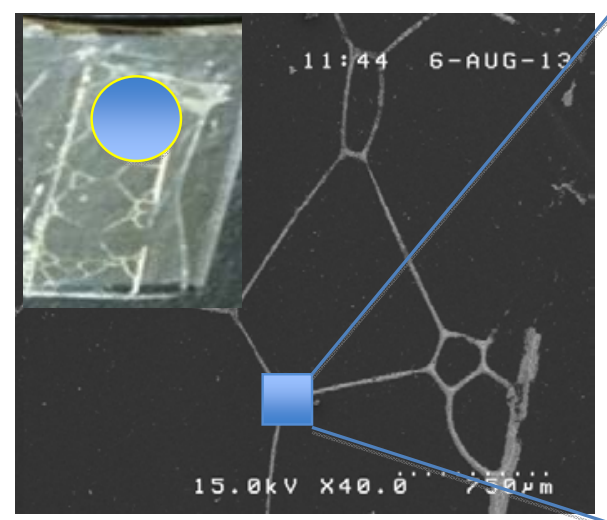

(a)

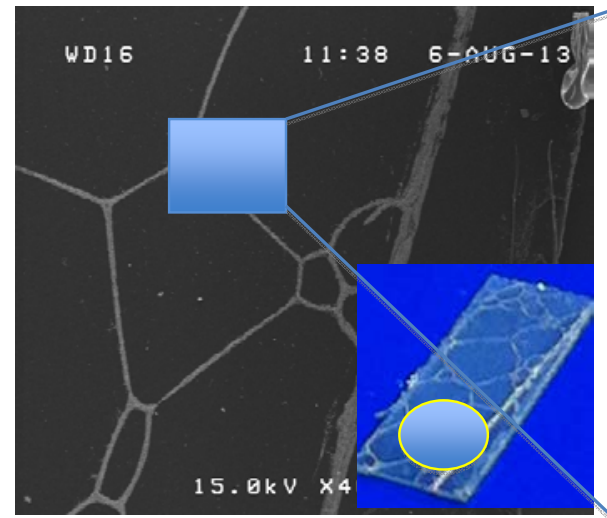

(c)

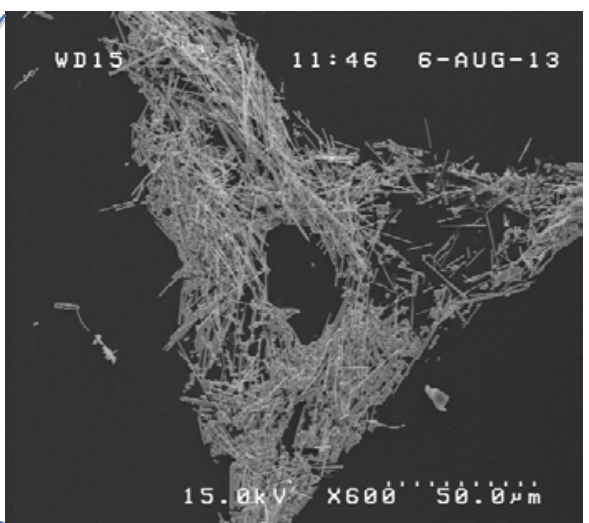

(b)

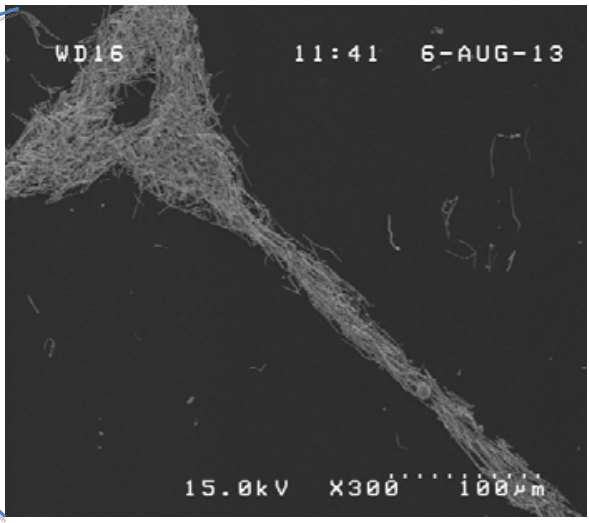

(d) 
Figure 5.

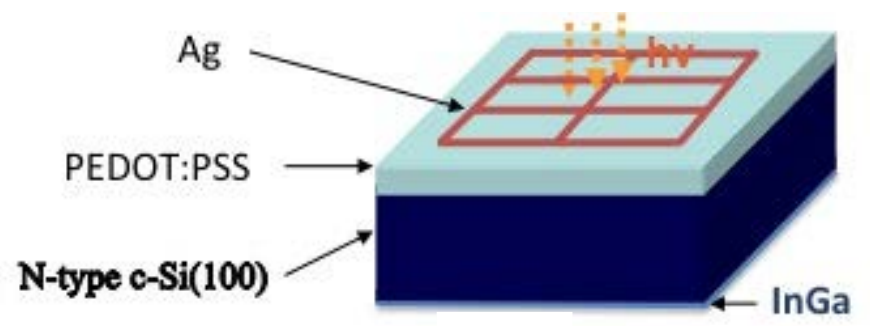

(a)

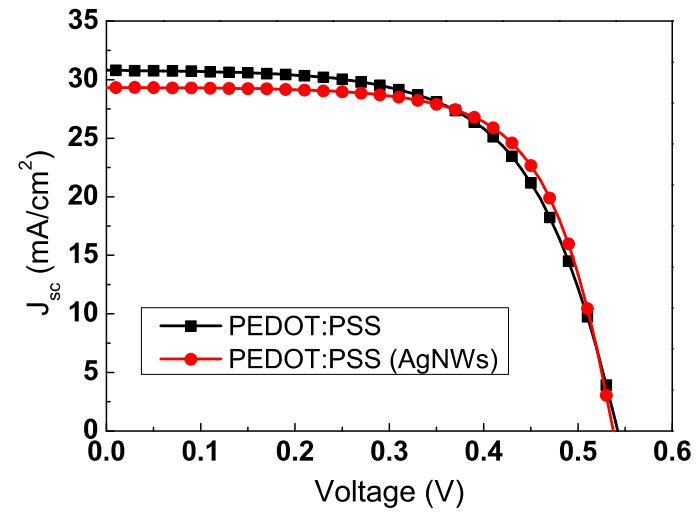

(b) 